\section{Long-term safety of deferiprone treatment in children from the Mediterranean region with beta-thalassemia major: the DEEP-3 multi-center observational safety study}

Early iron chelation therapy is fundamental in the treatment of $\beta$-thalassemia major ( $\beta$-TM) to avoid the adverse effects of chronic transfusional iron overload. Deferiprone (DFP) was the first oral iron chelator, but remains second line due to safety concerns. ${ }^{1}$ Superior efficacy in reducing cardiac iron, especially in combination with deferoxamine (DFO), has been shown. ${ }^{2}$ Its favourable cost-effectiveness and its off-patent status make it economically interesting both for developed and developing countries. Utilization in children is still limited since studies in pediatric patients are scarce.

The independent consortium Deferiprone Evaluation in Paediatrics (DEEP), born within the context of the TEDDY Network and funded by the EU Commission, investigates safety and efficacy of DFP. The DEEP-3 study investigated the long-term safety of DFP therapy alone and in combination with DFO.

DEEP-3 was a multi-center, retro- and prospective, non-interventional cohort study which was conducted in 16 hospitals in six Mediterranean countries between November 2012 and April 2016: Albania (1), Cyprus (1), Egypt (1), Greece (1), Italy (11) and Tunisia (1). Endpoints were the nature and incidence of serious and non-serious adverse drug reactions (ADR) to DFP, and potential risk factors for ADRs and DFP withdrawals.

Inclusion criteria were: diagnosis of $\beta$-TM and transfusional iron overload, start of DFP at age one month to less than 18 years, and at least one dose of DFP. Patients were excluded if previous medical records could not be obtained or were incomplete.

Start of observation was the initiation of DFP therapy. Follow up ended when the patient either completed the study (e.g., turned 18 years of age or end of study) or permanently withdrew from DFP for any reason (e.g., adverse reaction).

The study was approved by all local ethics committees. For prospective data collection, parents or guardians provided written informed consent.

The investigators were asked to report the following adverse events (AE): hospitalization, fever of unknown origin, thrombocytopenia (platelet count $<150 \times 10^{9} / \mathrm{L}$ ), neutropenia (neutrophil count $0.5-1.5 \times 10^{9} / \mathrm{L}$ ), agranulocytosis (neutrophil count $<0.5 \times 10^{9} / \mathrm{L}$ ), increased transaminases, arthropathies, neurological symptoms, hearing impairments, cardiac symptoms, endocrine dysfunctions, renal dysfunctions, weight gain $>5 \%$ of baseline, gastrointestinal disorders, cutaneous reactions, death, or any other clinically important events.

An independent safety committee evaluated causality, severity and seriousness of each AE. Disagreements were solved by consensus. Only AEs deemed at least possibly related to DFP by the committee and with positive investigator-reported causality were considered to be an ADR to DFP. Additional information on the methods can be found in the Online Supplementary File.

Two hundred and ninety-seven patients were enrolled and observed from March 1994 to October 2015, with 717.4 person-years (PY) of follow up (Table 1). Median patient age at start of DFP therapy was 8.5 years (range 0.6-17.6 years). One patient died from non-DFP-related vomitus aspiration and severe convulsions. Median follow up per patient was 1.7 years (IOR 0.8-3.5) and ranged from two days to 15.2 years. Further details are outlined in Online Supplementary Table S2.

Most patients received DFP monotherapy (68.3\%). Simultaneous therapy with DFO was predominate at Cyprus, Greek and Italian sites (Online Supplementary Table S3).

A total of 491 AEs including 158 serious AEs in 183 patients underwent the causality assessment (see full list in Online Supplementary Table S4). After assessment, 172 AE episodes in 104 patients were considered to be DFP related

Table 1. Baseline characteristics.

\begin{tabular}{|c|c|}
\hline Patients & $297(100.0)$ \\
\hline Age (years) & $8.5(4.0-12.2)$ \\
\hline$<6$ years & $112(37.7)$ \\
\hline $6-10$ years & $66(22.2)$ \\
\hline$>10$ years & $119(40.1)$ \\
\hline at diagnosis of $\beta$-TM & $0.8(0.4-1.2)$ \\
\hline at start of transfusion therapy & $0.7(0.5-1.2)$ \\
\hline at start of chelation therapy & $2.8(2.0-4.5)$ \\
\hline Sex & $297(100.0)$ \\
\hline Female & $145(48.8)$ \\
\hline Male & $152(51.2)$ \\
\hline Origin & $297(100.0)$ \\
\hline Europe & $131(44.1)$ \\
\hline North Africa & $154(51.9)$ \\
\hline Rest of Africa & $1(0.3)$ \\
\hline North America & $6(2.0)$ \\
\hline Latin America & $1(0.3)$ \\
\hline Asia & $4(1.4)$ \\
\hline$\beta$-TM type & $84(100.0)$ \\
\hline$\beta^{0} / \beta^{0}$ & $23(27.4)$ \\
\hline$\beta^{0} / \beta^{+}$ & $28(33.3)$ \\
\hline$\beta^{+} / \beta^{+}$ & $30(35.7)$ \\
\hline Hb Lepore $/ \beta^{0}$ & $1(1.2)$ \\
\hline Hb Lepore $/ \beta^{+}$ & $1(1.2)$ \\
\hline$\delta \beta / \beta^{+}$ & $1(1.2)$ \\
\hline Transfusional iron intake & $293(100.0)$ \\
\hline Low (<0.3 mg/kg/day) & $46(15.7)$ \\
\hline Intermediate (0.3-0.5 mg/kg/day) & $197(67.2)$ \\
\hline High (> 0.5 mg/kg/day) & $50(17.1)$ \\
\hline $\begin{array}{l}\text { Annual blood requirement } \\
\text { (mL pure RBC/kg/year) }\end{array}$ & $125(117-156)$ \\
\hline Spleen status & $289(100.0)$ \\
\hline Normal & $51(17.7)$ \\
\hline Splenomegaly & $168(58.1)$ \\
\hline Splenectomized & $70(24.2)$ \\
\hline Serum ferritin & $268(100.0)$ \\
\hline$<2,000$ ng/mL (n, \%) & $143(53.4)$ \\
\hline 2,000-4,000 ng/mL (n, \%) & $93(34.7)$ \\
\hline$>4,000$ ng/mL (n, \%) & $32(11.9)$ \\
\hline HIV seropositive $(\mathrm{n}=183)$ & $0(0.0)$ \\
\hline Hepatitis B seropositive $(\mathrm{n}=237$ ) & $10(4.2)$ \\
\hline Hepatitis $C$ seropositive $(n=242)$ & $41(16.9)$ \\
\hline
\end{tabular}

otherwise; b-TM: b-thalassemia major; RBC: red blood cells 
Table 2. Identified adverse drug reactions (ADR) to deferiprone (DFP).

\begin{tabular}{|c|c|c|c|c|c|c|}
\hline $\begin{array}{l}\text { MedDRA SOC / } \\
\text { Preferred Term }\end{array}$ & $\begin{array}{l}\text { ADRs / } \\
\text { Patients }\end{array}$ & $\begin{array}{c}\text { Severity } \\
\text { mild / } \\
\text { moderate / } \\
\text { severe }\end{array}$ & $\begin{array}{c}\text { Seriousness } \\
\text { non-serious / } \\
\text { serious }\end{array}$ & $\begin{array}{l}\text { Incidence }^{1} \\
\text { (95\% CI) }\end{array}$ & $\begin{array}{c}\text { Incidence } \\
\text { rate }^{2} \\
(95 \% \text { CI) }\end{array}$ & $\begin{array}{c}\text { DFP } \\
\text { discontinuation } \\
(95 \% \text { CI) }\end{array}$ \\
\hline \multicolumn{7}{|c|}{ Blood and lymphatic system disorders } \\
\hline Agranulocytosis & $2 / 2$ & $0 / 2 / 0$ & $0 / 2$ & $0.7(0.1-2.4)$ & $0.3(0.0-1.0)$ & $0.7(0.1-2.4)$ \\
\hline Leukopenia & $3 / 2$ & $3 / 0 / 0$ & $3 / 0$ & $0.7(0.1-2.4)$ & $0.4(0.1-1.2)$ & $0.3(0.0-1.9)$ \\
\hline Neutropenia & $38 / 25$ & $26 / 12 / 0$ & $0 / 38$ & $8.4(5.5-12.2)$ & $5.3(3.7-7.3)$ & $5.1(2.9-8.2)$ \\
\hline Thrombocytopenia & $1 / 1$ & $1 / 0 / 0$ & $0 / 1$ & $0.3(0.0-1.9)$ & $0.1(0.0-0.8)$ & $0.3(0.0-1.9)$ \\
\hline \multicolumn{7}{|l|}{ Gastrointestinal disorders } \\
\hline Abdominal pain & $9 / 8$ & $6 / 3 / 0$ & $9 / 0$ & $2.7(1.2-5.2)$ & $1.3(0.6-2.4)$ & $1.0(0.2-2.9)$ \\
\hline Diarrhea & $1 / 1$ & $1 / 0 / 0$ & $1 / 0$ & $0.3(0.0-1.9)$ & $0.1(0.0-0.8)$ & none \\
\hline Dyspepsia & $4 / 4$ & $4 / 0 / 0$ & $4 / 0$ & $1.3(0.4-3.4)$ & $0.6(0.2-1.4)$ & $0.3(0.0-1.9)$ \\
\hline Nausea & $3 / 3$ & $1 / 2 / 0$ & $3 / 0$ & $1.0(0.2-2.9)$ & $0.4(0.1-1.2)$ & $0.3(0.0-1.9)$ \\
\hline Salivary hypersecretion & $1 / 1$ & $1 / 0 / 0$ & $1 / 0$ & $0.3(0.0-1.9)$ & $0.1(0.0-0.8)$ & none \\
\hline Vomiting & $14 / 10$ & $3 / 11 / 0$ & $14 / 0$ & $3.4(1.6-6.1)$ & $2.0(1.1-3.3)$ & $2.7(1.2-5.2)$ \\
\hline
\end{tabular}

General disorders and administration site conditions

\begin{tabular}{|c|c|c|c|c|c|c|}
\hline Fatigue & $1 / 1$ & $0 / 1 / 0$ & $1 / 0$ & $0.3(0.0-1.9)$ & $0.1(0.0-0.8)$ & $0.3(0.0-1.9)$ \\
\hline \multicolumn{7}{|l|}{ Investigations } \\
\hline Transaminases increased & $42 / 31$ & $35 / 7 / 0$ & $17 / 25$ & $10.4(7.2-14.5)$ & $5.9(4.2-7.9)$ & $6.4(3.9-9.8)$ \\
\hline Weight increased & $1 / 1$ & $1 / 0 / 0$ & $1 / 0$ & $0.3(0.0-1.9)$ & $0.1(0.0-0.8)$ & none \\
\hline \multicolumn{7}{|c|}{ Musculoskeletal and connective tissue disorders } \\
\hline Arthropathy & $43 / 35$ & $10 / 30 / 3$ & $41 / 2$ & $11.8(8.3-16.0)$ & $6.0(4.3-8.1)$ & $8.4(5.5-12.2)$ \\
\hline Bone pain & $5 / 5$ & $2 / 3 / 0$ & $5 / 0$ & $1.7(0.5-3.9)$ & $0.7(0.2-1.6)$ & $1.3(0.4-3.4)$ \\
\hline \multicolumn{7}{|l|}{ Renal and urinary disorders } \\
\hline Chromaturia & $1 / 1$ & $1 / 0 / 0$ & $1 / 0$ & $0.3(0.0-1.9)$ & $0.1(0.0-0.8)$ & none \\
\hline \multicolumn{7}{|c|}{ Skin and subcutaneous tissue disorders } \\
\hline Rash & $2 / 1$ & $2 / 0 / 0$ & $2 / 0$ & $0.3(0.0-1.9)$ & $0.3(0.0-1.0)$ & none \\
\hline Urticaria & $1 / 1$ & $0 / 1 / 0$ & $1 / 0$ & $0.3(0.0-1.9)$ & $0.1(0.0-0.8)$ & none \\
\hline \multicolumn{7}{|l|}{ TOTAL } \\
\hline Any ADR & $172 / 104$ & $97 / 72 / 3$ & $104 / 68$ & $35.0(29.6-40.7)$ & $24.0(20.5-27.8)$ & $23.2(18.6-28.5)$ \\
\hline Any serious ADR & $68 / 44$ & $48 / 19 / 1$ & $0 / 68$ & $14.8(11.0-19.4)$ & $9.5(7.4-12.0)$ & $9.1(6.1-13.0)$ \\
\hline
\end{tabular}

${ }^{1}$ Number of patients with at least one ADR from the corresponding group divided by all exposed patients in percent (95\% CI); ${ }^{2} \mathrm{Number}$ of ADR episodes divided by total observation time per $100 \mathrm{PY}(95 \% \mathrm{CI}) ;{ }^{3}$ Number of patients permanently discontinuing DFP due to the ADR divided by all exposed patients in percent (95\% CI). PY: personyears; CI: confidence interval; MedDRA SOC: MedDRA System Organ Class.

ADRs (Table 2). The incidence and incidence rate of serious ADRs was $14.8 \%$ and 9.5 per 100 PY, respectively. The probability of experiencing a serious ADR was $20.6 \%$ after 36 months of treatment (data not shown). ADR incidence did not differ significantly between patients using DFP products Ferriprox or Kelfer $(P=0.491)$.

DFP-related neutropenia was identified in 25 nonsplenectomized patients (incidence 8.4\%). There was no difference in neutropenia incidence between normal spleen size and splenomegaly $(P=0.737)$. In three patients, neutropenia was recurrent despite temporary termination and DFP was eventually discontinued.

Agranulocytosis occurred in two female patients in the first 12 months of treatment without previous neutropenia (Figure 1) and recovered upon DFP discontinuation (incidence $0.7 \%$ ); one patient was on combined therapy and one on monotherapy. Both patients received standard DFP and DFO doses and had splenomegaly with normal neutrophil levels at baseline. A third case of agranulocytosis was demonstrated to be parvovirus B19 related.

Gastrointestinal disorders, e.g., abdominal pain, diarrhea, dyspepsia, or nausea, were observed with incidences ranging between $0.3 \%$ and $3.4 \%$. None of these $\mathrm{ADRs}$ were serious. Median time to occurrence was three months (IOR 0.0-9.0).

Increased transaminases were seen in 31 patients (incidence $10.4 \%$ ). The respective alanine transaminase levels were as follows: two ADRs $<2 \mathrm{x}$ upper limit of normal (ULN), 24 ADRs 2-5x ULN, 14 ADRs 5-10x ULN, and two ADRs $>10 \mathrm{x}$ ULN. There was no difference between HCV positive and negative patients $(P=0.549)$. Median time to first occurrence was eight months (IOR 4-23).

Arthropathy, including arthralgia and swelling, mostly in the knee joints, was the most common $\mathrm{ADR}$ (Table 2). In five patients, severe and disabling symptoms occurred. More than half of the affected patients experienced symptoms in the first 12 months of treatment (Figure 1). North African patients $(24.2 \%)$ were more often affected than European patients $(3.8 \%)(P<0.001)$.

Other ADRs identified included mild rash and urticaria, moderate fatigue, mild weight increase, and mild chromaturia. No drug-related worsening of hepatic fibrosis, audio- 


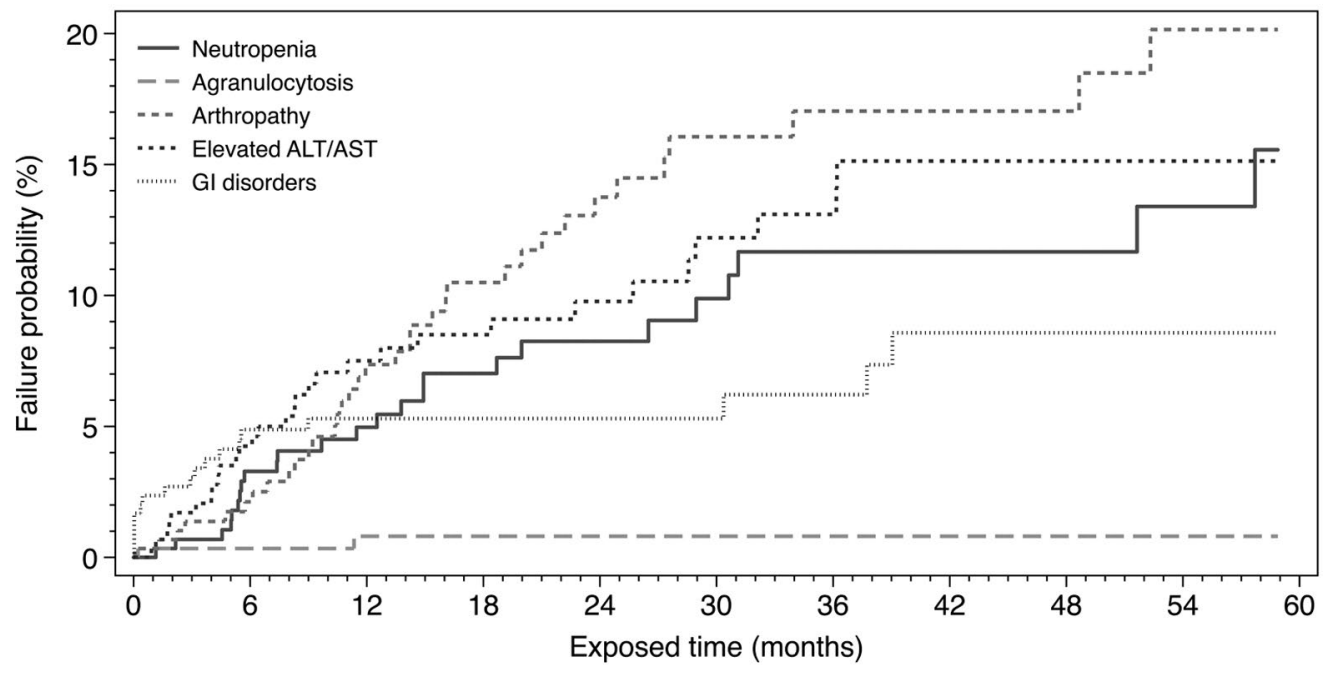

$\begin{array}{rlllllllllllllllllllll}\text { Neutropenia } & 297 & (9) & 258 & (4) & 197 & (4) & 158 & (2) & 124 & (2) & 103 & (2) & 86 & (0) & 71 & (0) & 58 & (1) & 46 & (1) & 35 \\ \text { Agranulocytosis } & 297 & (1) & 262 & (1) & 202 & (0) & 162 & (0) & 129 & (0) & 108 & (0) & 91 & (0) & 75 & (0) & 61 & (0) & 49 & (0) & 38 \\ \text { Arthropathy } & 297 & (6) & 257 & (12) & 195 & (6) & 152 & (5) & 121 & (3) & 100 & (1) & 83 & (0) & 70 & (0) & 57 & (2) & 47 & (0) & 37 \\ \text { Elevated ALT/AST } & 297 & (12) & 257 & (8) & 196 & (2) & 158 & (2) & 124 & (3) & 102 & (1) & 86 & (2) & 69 & (0) & 55 & (0) & 44 & (0) & 34 \\ \text { GI disorders } & 297 & (14) & 253 & (1) & 197 & (0) & 160 & (0) & 127 & (0) & 106 & (1) & 88 & (2) & 70 & (0) & 56 & (0) & 46 & (0) & 36\end{array}$

Figure 1. Kaplan-Meier failure analysis. Values are number of patients at risk at the corresponding time point and in brackets the number of deferiprone-related adverse drug reactions during the respective 6-month period (only first occurrence considered per patient). Censored observation markers and exposure after 60 months have been omitted for the sake of curve simplicity.

logical disorders, visual toxicities or renal impairments were observed. Adverse reactions disappeared upon temporary DFP interruption, dose reduction or even without intervention.

DFP was discontinued due to AEs (52.9\%), lack of efficacy $(17.0 \%)$ and non-compliance $(7.8 \%)$. Online Supplementary Tables $S 5$ and $S 6$ provide more details.

In $23.2 \%$ of patients, an ADR to DFP led to permanent therapy discontinuation, most commonly due to arthropathy, increased transaminases, neutropenia, and gastrointestinal disorders (Table 1). There were significantly more ADR-related discontinuations in children under the age of 10 years than in older children $(P=0.025)$. No specific risk factors for $\mathrm{ADR}$ or DFP withdrawals could be identified (Online Supplementary Table S7).

DEEP-3 was the largest safety study of DFP in children so far with more than half of our patients below the age of 10 years, and more than one-third below six years.

Compared to adults, neutropenia seems to occur more often in children, even healthy ones. ${ }^{3}$ However, our agranulocytosis and neutropenia incidences are similar to those reported in children and adolescents on DFP. ${ }^{4}$ The association between neutropenia and DFP remains uncertain. Our and other studies showed that patients recovered despite DFP continuation, or did not have neutropenia on re-challenge. ${ }^{5-7}$ Splenectomy may protect from neutropenia as only non-splenectomized patients were affected from neutropenia and agranulocytosis. Similar observations have been reported before. ${ }^{1,8,9}$ Ethnic differences may influence the occurrence of agranulocytosis as reported in Chinese children on DFP. ${ }^{10}$ Combined therapy was not associated with a higher risk for neutropenia consistent with existing data. ${ }^{4}$ European sites reported neutropenia more often than non-European sites ( $13.5 \%$ vs. $4.3 \%, P=0.004)$ due to closer neutrophil monitoring.

For agranulocytosis, we can confirm the following obser- vations: it is most likely seen in the first 12 months of treatment, more often in female patients, and independent from dose and combination with $\mathrm{DFO} .^{3}$ The rare occurrence did not allow the investigation of potential risk factors. In literature, it is depicted to be idiosyncratic, unpredictable and not dose-dependent. ${ }^{4}$

Arthropathy incidence is in agreement with previous studies. ${ }^{4}$ We could not find a correlation between high serum ferritin (SF) and arthropathy, but we found significantly more cases in North African patients. There may be ethnic differences similar to the high incidences reported in Indian children. ${ }^{5,11,12}$ We cannot confirm the influence of different DFP products on the occurrence of arthropathy. ${ }^{8,13}$

Elevated transaminases were the second most frequently identified ADR to DFP. We could not find an association between the adverse reaction and $\mathrm{HCV}$ serostatus or SF levels, respectively.

Gastrointestinal disorders affected $6.4 \%$ of the patients which is lower than reported in previous pediatric studies with incidences between $11 \%$ and $27.3 \%$. ${ }^{4}$ The actual incidence may be higher because we must take underreporting of mild events into account.

DFP discontinuation rate was $23.2 \%$ in our pediatric cohort. Other observational studies involving real-world data reported rates of $8.8 \%$ and $29.3 \%{ }^{14,15}$ ADR-related DFP discontinuations were more common in children under the age of 10 years. It may be more difficult to readminister a drug associated with burdensome effects in younger children.

In conclusion, we did not find any unexpected ADRs, and incidences are in accordance with available data. Most reactions were mild or moderate and patients recovered shortly upon dose reduction, temporary interruption or withdrawal. Neither patients under the age of 6 years, nor under 10 years, experienced more ADRs than older patients. We also did not find a greater risk for ADRs in 
patients on combined iron chelation therapy with DFO.

However, agranulocytosis was also present in this pediatric population. Weekly monitoring of neutrophils is warranted. Arthropathy has significant impact on quality of life in children and needs to be closely monitored. A new ageappropriate oral formulation of DFP developed in the DEEP project may improve gastrointestinal tolerability. Further efforts should be made to facilitate the availability of affordable, safe and effective iron chelation for children worldwide.

Sebastian Botzenhardt, ${ }^{1}$ Mariagrazia Felisi, ${ }^{2}$ Donato Bonifazi, Giovanni C. Del Vecchio, ${ }^{3}$ Maria C. Putti, ${ }^{4}$

Antonis Kattamis, ${ }^{5}$ Adriana Ceci, ${ }^{2,6}$ Ian C.K. Wong ${ }^{7,8}$ and

Antje Neubert ${ }^{\prime}$ on behalf of the DEEP consortium (collaborative group)

Department of Paediatrics and Adolescent Medicine, Faculty of Medicine, Friedrich-Alexander University Erlangen-Nürnberg (FAU), Erlangen, Germany; ${ }^{2}$ Consorzio per Valutazioni Biologiche e Farmacologiche, Pavia, Italy; ${ }^{3}$ Azienda Ospedaliera Universitaria Consorziale, Policlinico di Bari, Italy; ${ }^{4}$ Azienda Ospedaliera di Padova, Clinica di Oncoematologia Pediatrica, Italy; ${ }^{5}$ First Department of Pediatrics, National and Kapodistrian University of Athens, Greece; ${ }^{6}$ Gianni Benzi Pharmacological Research Foundation, Bari, Italy; ${ }^{7}$ Centre for Medicines Optimisation Research and Education, Research Department of Practice \& Policy, University College London School of Pharmacy, UK and ${ }^{\circ}$ Centre for Safe Medication Practice and Research, Department of Pharmacology and Pharmacy, Li Ka Shing Faculty of Medicine, The University of Hong Kong, China

Funding: the research leading to these results has received funding from the European Union's Seventh Framework Programme (FP7) under grant agreement no 261483 (Deferiprone Evaluation in Paediatrics, DEEP).

Acknowledgments: the authors would like to thank all participating patients, investigators and study staff of the whole DEEP consortium for their tremendous work and efforts without this study couldn't have been made possible. Cristina Manfredi and Rosa Padula remarkably managed the correspondence with Italian regulatory authorities and Italian sites. Luca Forlani is acknowledged for his efforts with the eCRF implementation. Beatrice Pantaleo, Simona Ravera and Francesca Borgia are thanked for their significant work in the ADR assessment. Great commitment of the following investigators must be highlighted: Amal ElBeshlawy, Mona El-Taqui, Amina Abdel-Salam, Naglaa Omar, Khaled Eid, Mona El-Ghamrawy, Sonia Adolf, Mai Saad (Egypt); Aldo Filosa, Carlo Cosmi, Aurelio Maggio, Raffaela Origa (Italy); Soteroula Christou, Evi Missouri (Cyprus); Manika Kreka, Eleni Nastas (Albania); Monia Romdhane, Mohamed Bejaoui (Tunis)

Participating sites and principle investigators. Cairo University, Egypt (Amal El-Beshlawy); National and Kapodistrian University of Athens, Greece (Antonis Kattamis); Qendra Spitalore Universitare Nene Tereza, Tirana, Albania (Eleni Nastas); Cyprus Ministry of Health, Nicosia Thalassaemia Center, Cyprus (Soteroula Christou); Centre national de Greffe de Moelle Osseuse, Tunis, Tunisia (Mohamed Bejaoui); Azienda Ospedaliera di Rilievo Nazionale Antonio Cardarelli, Naples, Italy (Aldo Filosa); Azienda Ospedaliera Ospedali Riuniti Villa Sofia-Cervello, Palermo, Italy (Aurelio Maggio); Azienda Ospedaliera di Padova, Padua, Italy (Maria Caterina Putti); Azienda Ospedaliero-Universitaria Consorziale Policlinico di Bari, Italy (Giovanni Carlo Del Vecchio); ARNAS-Civico G. di CristinaBenfratelli, Palermo, Italy (Liana Cuccia); Azienda Ospedaliera di Cosenza, Italy (Mariagrazia Bisconte); Ospedale Civile di Lentini,
Italy (Francesca Commendatore); Policlinico di Modena, Modena, Italy (Giovanni Palazzi); Azienda Mista Ospedaliera-Universitaria di Sassari, Sassari, Italy (Carlo Cosmi); Ospedale Pediatrico Microcitemico "A.Cao", University of Cagliari, Italy (Raffaella Origa); Azienda Ospedaliero-Universitaria Meyer, Florence, Italy (Tommaso Casini).

Correspondence: antje.neubert@uk-erlangen.de doi:10.3324/haematol.2017.176065

Information on authorship, contributions, and financial \& other disclosures was provided by the authors and is available with the online version of this article at www. haematologica.org.

\section{References}

1. Cohen AR, Galanello R, Piga A, De Sanctis V, Tricta F. Safety and effectiveness of long-term therapy with the oral iron chelator deferiprone. Blood. 2003;102(5):1583-1587.

2. Tanner MA, Galanello R, Dessi C, et al. A randomized, placebo-controlled, double-blind trial of the effect of combined therapy with deferoxamine and deferiprone on myocardial iron in thalassemia major using cardiovascular magnetic resonance. Circulation. 2007;115(14):1876-1884

3. Tricta F, Uetrecht J, Galanello R, et al. Deferiprone-induced agranulocytosis: 20 years of clinical observations. Am J Hematol. 2016;91(10):1026-1031.

4. Botzenhardt S, Li N, Chan EW, Sing CW, Wong IC, Neubert A. Safety profiles of iron chelators in young patients with haemoglobinopathies. Eur J Haematol. 2017;98(3):198-217.

5. Choudhry VP, Pati HP, Saxena A, Malaviya AN. Deferiprone, efficacy and safety. Indian J Pediatr. 2004;71(3):213-216.

6. El-Beshlawy AM, El-Alfy MS, Sari TT, Chan LL, Tricta F. Continuation of deferiprone therapy in patients with mild neutropenia may not lead to a more severe drop in neutrophil count. Eur J Haematol. 2014;92(4):337-340.

7. Elalfy M, Wali YA, Qari M, et al. Deviating from safety guidelines during deferiprone therapy in clinical practice may not be associated with higher risk of agranulocytosis. Pediatr Blood Cancer. 2014;61(5):879-884.

8. Viprakasit V, Nuchprayoon I, Chuansumrit A, et al. Deferiprone (GPO-L-ONE $®$ ) monotherapy reduces iron overload in transfusiondependent thalassemias: 1-year results from a multicenter prospective, single arm, open label, dose escalating phase III pediatric study (GPO-L-ONE; A001) from Thailand. Am J Hematol. 2013;88(4):251260

9. ElAlfy MS, Sari TT, Lee CL, Tricta F, El-Beshlawy A. The safety, tolerability, and efficacy of a liquid formulation of deferiprone in young children with transfusional iron overload. J Pediatr Hematol Oncol. 2010;32(8):601-605.

10. Botzenhardt S, Sing CW, Wong IC, et al. Safety profile of oral iron chelator deferiprone in chinese children with transfusion-dependent thalassaemia. Curr Drug Saf. 2016;11(2):137-144.

11. Agarwal MB, Gupte SS, Viswanathan C, et al. Long-term assessment of efficacy and safety of L1, an oral iron chelator, in transfusion dependent thalassaemia: Indian trial. Br J Haematol. 1992;82(2):460466.

12. Sanjeeva GN, Nijaguna N, Mahantesh M, Pooja Gujjal C. Efficacy and safety of deferasirox when compared to deferiprone as oral iron chelating agent: a randomized control trial. J of Evolution of Med and Dent Sci. 2015;4(24):4178-4185

13. Viprakasit V, Rodmai S, Srichairatanakool S. Deferiprone for transfusional iron overload and its roles in developing countries. Expert Opin Orphan Drugs. 2014;2(2):189-200.

14. Ceci A, Baiardi P, Felisi M, et al. The safety and effectiveness of deferiprone in a large-scale, 3-year study in Italian patients. Br J Haematol. 2002;118(1):330-336

15. Borgna-Pignatti C, Cappellini MD, De Stefano P, et al. Cardiac morbidity and mortality in deferoxamine- or deferiprone-treated patients with thalassemia major. Blood. 2006;107(9):3733-3737. 